\title{
Multi-functional coating of polymeric spherulites for chiral photonic cellulose nanocrystal films
}

\author{
Zahra Bazrafshan $(\mathbb{D}$
}

Received: 2 January 2020/ Accepted: 11 May 2020/Published online: 25 May 2020

(C) The Author(s) 2020

\begin{abstract}
Cellulose Nanocrystal (CNC) films are from emerging nanoparticles recently known for photonic properties. The CNC films show nonuniform photonic domains resulting from structural irregularities in their typical self-assembled arrangements. This paper presents a drop-casting approach from Polyamide 6 to form a coating layer on the CNC films. This coating approach led to polycrystalline growth patterns known as spherulites on the CNC film. For the resulting coated $\mathrm{CNC}$ film, the spherulites yielded a unique set of enhanced physical properties including optical, mechanical and thermal as well as a new morphological appearance from their micro-scale average spherical shape; the ordering effect of their local radial symmetry and orientation positively influenced the effects of structural irregularities of the typical CNC films. Under polarized light, the optical transmission bands of the CNC film were perceived through radial symmetry of banded spherulites which were responsible for the formation of circular extinction patterns for the coated CNC film. The intermolecular interactions and interlamellar entanglement of spherulites provided enhanced flexibility and tensile strength for the coated CNC film. This protecting design allows for the future
\end{abstract}

Z. Bazrafshan $(\bowtie)$

School of Textiles and Design, Heriot-Watt University, Galashiels, UK

e-mail: z.bazrafshan@hw.ac.uk;

zahrabazrafshan@yahoo.com development of a variety of portable functional assemblies requiring enhanced performance in optical, mechanical and thermal properties of $\mathrm{CNC}$ films.

Keywords Chiral photonic cellulose nanocrystal film - Spherulites · Polyamide 6 - Optical properties . Mechanical reinforcement

\section{Introduction}

Cellulose and particularly cellulose nanocrystals, as a sustainable bio-based material, have recently enabled new venues in the fabrication of photonic components used, for example, in chiral reflectors (Dumanli et al. 2014a), photonic electrodes (Habibi et al. 2008), antireflection coatings for photovoltaics (Tsui et al. 2014), and humidity responsive optical materials (Yao et al. 2017). Optical properties of cellulose nanocrystals $(\mathrm{CNCs})$ are evaluated in the preparation and characterization of iridescent films through solvent evaporation of sulfate cellulose nanocrystals suspensions in polar solvents, e.g., water. Several researchers have shown that dried CNC films display outstanding optical activity including circular dichroism and Bragg reflection (Peng et al. 2011; Diddens et al. 2008; Zhang et al. 2013).

Retention of self-assembled microstructures typically results in nonuniform photonic $\mathrm{CNC}$ films whose 
optical properties depend on different factors initiated from amorphous and varied helix orientation degree and pitch length of CNCs arrangements (Saha and Davis 2018; Kelly et al. 2013). These non-uniformities are usual of any self-assembly methods which can simply result in defect-rich with irregular mosaic-like multi-colored domains. For certain applications required flexibility and mechanical strength, thin photonic CNC films have also shown limited flexibility, brittleness leading to unfavorable mechanical behavior (Zhu et al. 2019; Sinko et al. 2013).

To overcome these challenges, several strategies have been proposed recently to control film formation based on helix orientation or the surface modification of CNCs. For instance, Wilts et al. achieved relatively uniform photonic films by controlling over film formation itself; helix orientation through slowly drying CNC dispersions in a custom-built humiditycontrolled chamber (Wilts et al. 2017). In a similar work, Saha and Davis considered extra process parameters such as CNC concentration, orbital shear, surface anchoring influencing CNC film formation (Saha and Davis 2018). By contrast, several surface modifications carried out with the presence of compatible ions (Liu et al. 2017) or low molecular weight polyelectrolytes (Yao et al. 2017; Xu et al. 2018) normalizing interparticle forces associated with film casting and shrinkage (Viet et al. 2007; Samir et al. 2004). In a mechanical reinforcement approach, low molecular weight hydrophilic polymers as plasticizers are also investigated to maintain the integrity, to improve the flexibility and handling of films, and to avoid pores and cracks during gelation and film formation (Samir et al. 2004). This is despite the fact that any surface modification method can be potentially along with by-products requiring extra purification steps.

However, achieving flexible arrangements from pristine $\mathrm{CNCs}$ has obtained some interesting results from post-treatment methods. In literature, the authors have reported approaches to covalently attach polymeric chains to the top layer of CNC films (Alanis et al. 2019). These techniques are still very limited in terms of scalability, compatibility, and reproducibility because they usually use hydrophobic materials including polymers, monomers, and initiators that may feel considerable steric hindrance to access the reaction site on $\mathrm{CNC}$ films, possibly resulting in further non-uniformities due to varied grafting densities on the surface (Bazrafshan and Stylios 2019).

A coating approach can be another possible posttreatment to $\mathrm{CNC}$ films. The coating techniques are widely studied for inorganic/organic photonic/semiconducting materials to control optical, mechanical and electrical properties (Eggleton et al. 2011; Yang et al. 2018). However, they have not been used for CNC films considering enhanced physicochemical properties that are induced by coating materials. This work presents a drop-casting approach from Polyamide 6 (PA6) to form a coating layer on CNC films. This coating approach led to polycrystalline growth patterns known as spherulites on the CNC film. The evident similarities of their morphologies and tendency for space-filling (of potential pores and cracks of CNC films as a coating/protecting layer), polycrystallinity, and their elongated fiber-like grains stimulated the hypothesis of this work that this type of pattern formation can positively influence the impact of structural irregularities of typical CNC films which were formed under ambient conditions. The practical importance of spherulite formation was considered including the crystal growth mechanism and the effect of their structures on the physical properties of CNC films such as optical, mechanical and thermal. This experimental work can be potentially used for optical sensors, anti-counterfeiting industries, and decorations. Since a large variety of semicrystalline polymers is available, we expect that spherulites occur in the same way from other polymers under their thermodynamic conditions, and this surface design and coating approach is suggested for the future development of a variety of portable functional assemblies of CNC films for photonic sensing requiring enhanced performance in optical and mechanical properties.

\section{Experimental}

Materials

$\alpha$-Cellulose (powder) and nylon 6 (PA6, pellet) were supplied by Sigma-Aldrich and sulfuric acid (98\%) and Formic Acid (99\%) was prepared by Alfa Aesar. Distilled water was used for dilution and purification of CNCs. 
Preparation of CNC suspension and film

CNC suspension was prepared from $\alpha$-Cellulose by sulfuric acid hydrolysis (Bondeson et al. 2006; Sheltami et al. 2012). Briefly, CNC hydrolysis was carried out with sulfuric acid (64\%, wt./wt.) at $45{ }^{\circ} \mathrm{C}$ for $60 \mathrm{~min}$ with vigorous stirring and then it was diluted five times to stop the hydrolysis reaction. The micro-sized components were removed by filtration. The other possible monocrystalline components remained. After removing the acid, dynamic light scattering analysis (DLS, Malvern Zetasizer Nano ZS equipment) was performed on diluted suspensions in $0.01 \mathrm{wt} \%$ at $25{ }^{\circ} \mathrm{C}$. The rod-like shape and size of the CNCs in nanoscale were also studied by SEM analysis. For CNC film formation, freshly prepared CNC suspension $(20 \mathrm{ml})$ having an approximate solid content of $1 \mathrm{wt} \%$ in distilled water, where the $\mathrm{pH}$ of $\mathrm{CNC}$ suspension was around 5 at the end of the preparation procedure, was placed in an open Petri dish $(100 * 150 \mathrm{~mm})$. The cellulose suspensions were left for slow evaporation at room temperature for $72 \mathrm{~h}$ to obtain solidified CNC films.

\section{Coating of CNC film}

The dried films supported by the Petri dishes were covered and cooled for half an hour to reach the temperature of $\left(-10^{\circ} \mathrm{C}\right)$ and then sited on a cubic icebox. A solution of PA6 $(20 \mathrm{wt} / \mathrm{v} \%)$ in formic acid was heated up to a temperature of $70{ }^{\circ} \mathrm{C}$ (above glass transition temperature). The surface of the CNC film was coated by a $2 \mathrm{ml}$ PA6 solution through the needle of a syringe at a temperature of $20 \pm 2{ }^{\circ} \mathrm{C}$ and a humidity of $32 \%$, as shown schematically in Fig. 1 and left disrupted at room temperature for $10 \mathrm{~h}$ to be thoroughly hardened. The coated $\mathrm{CNC}$ film was simply detached at once from the petri dish for analyzing.

\section{Optical microscopy}

A Yenway CX40-POL microscope was used to capture all the polarized and non-polarized reflected light micrographs using $20 \times / 0.45,50 \times / 0.55$ and $100 \times / 0.95$ LM Plan objectives. The camera used was a YenCam ISH500 and the sample films were placed directly on glass slides, for some specific illustrations on polystyrene sheets as further explained in the "Results" section. The color histograms based on captured images were achieved using color image processing of ImageJ in where the brightnessweighted average of the $\mathrm{x}$ and $\mathrm{y}$ coordinates all pixels in the images. The colors in RGB images (24-bit with 8-bits for blue channels) were used to characterize colorful images. The associated color histograms were provided to reflect the brightness diversity of blue color in blue channels, which reflects the quality of this color in the specimen.

\section{Scanning electron microscopy}

A Hitachi S-4300 scanning electron microscope was used for SEM analysis using a $3 \mathrm{kV}$ electron beam
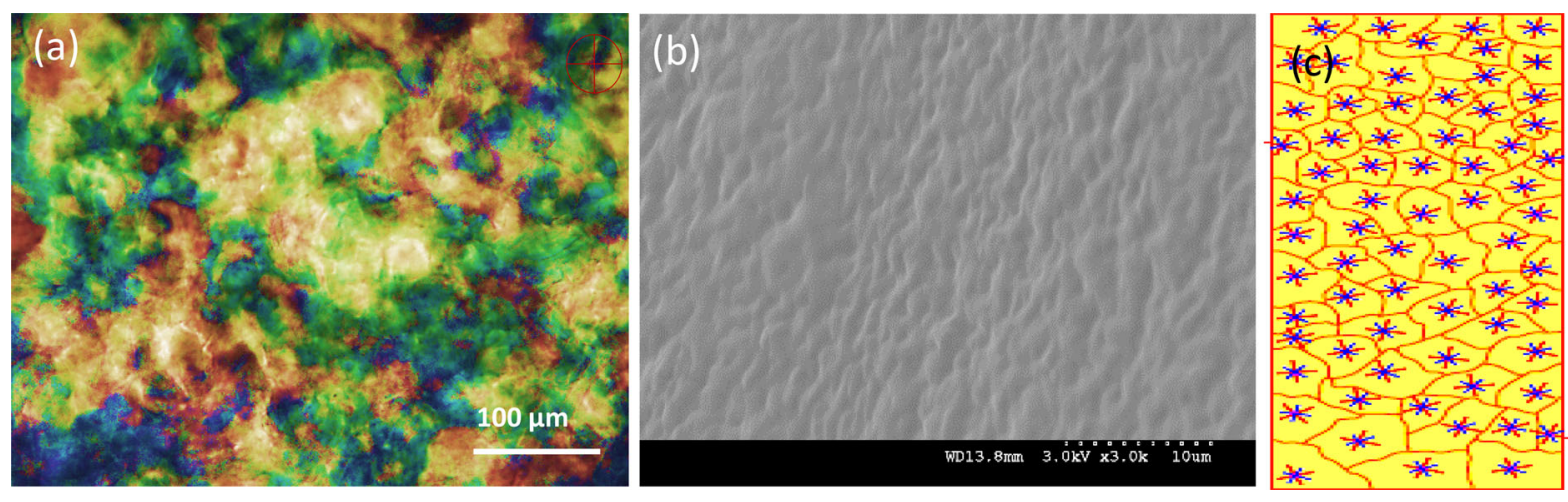

Fig. 1 A typical surface of an air-dried iridescent CNC film showing non-uniformities, irregular mosaic-like colorful domainsas a result of a non-assisted film formation process (no clear fingerprint texture was perceived in this scale); $\mathbf{a}$ cross- polarizedimage, b SEM image, c Sketches of irregular multi colorful domains that can be varied in brightness even in each domain 
voltage. Before SEM, gold-platinum sputtering was applied to the typical and coated CNC films run by Argon for plasma sputter. To study the shape and approximate size of the CNCs, approximately $0.5 \mathrm{~mL}$ of $0.01 \mathrm{wt} \% \mathrm{CNC}$ dispersion was drop cast onto a polyester membrane. The dispersion was allowed to sit for 5 min followed by air drying to observe CNCs on the surface. Then, the CNCs was sputtered by goldplatinum before doing SEM analysis. The shape and size measurements on SEM images was performed by ImageJ software.

UV-vis absorption/reflection and Fouriertransform infrared spectroscopy

A Perkin-Elmer ultraviolet-visible spectrometer (Lambda 35) was used to record the UV-vis spectra of samples. The optical reflectance of the sample films was measured over the wavelength region from 200 to $1100 \mathrm{~nm}$ under a $90^{\circ}$ incident illumination. To record the reflectance spectra, Labsphere reflectance spectroscopy accessories (RSA-PE) were used. A Nicolet iS5 FTIR spectrometer as used for Fourier-transform infrared (FTIR) assessments using the attenuated total reflectance (ATR) technique on dried typical and coated CNC film samples.

Thermogravimetric and Differential Scanning Calorimetry analyzer

A Mettler Tc 10A/TC15 analyzer was used for thermogravimetric analysis (TGA) of samples and a Mettler DSC 12E thermal analyzer was used to differential scanning calorimetry (DSC). Approximately $8 \mathrm{mg}$ dried film samples were subjected to a heating ramp from 25 to $450{ }^{\circ} \mathrm{C}$, at $10{ }^{\circ} \mathrm{C}$ increments and nitrogen flow of $50 \mathrm{~mL} \mathrm{~min}{ }^{-1}$.

Tensile test

Tensile testing as a fundamental test was performed using an Instron (3345 series) under controlled loading and tension on mounted specimens $(n=05$ of each sample) in the tester before subjecting each to tension using the BS-EN-ISO 527:2009 standard. The gauge length was set at $50 \mathrm{~mm}$ for all specimens and a rate of extension of $10 \mathrm{~mm} / \mathrm{min}$ under standard atmospheric conditions for testing $\left(23 \pm 1{ }^{\circ} \mathrm{C} ; 60 \pm 2 \% \mathrm{RH}\right)$. The tensile data collected, based on the extent of elongation and in the area of each specimen $(90$ $\mathrm{mm} * 15 \mathrm{~mm}$ ) as a function of load, tension and time, in which the tensile load is recorded along with increasing gauge length under a $10 \mathrm{~N}$ load cell. The stressstrain curve is plotted for each group of specimens. The advantage of stress-strain curves, in contrast with load-extension curves, is that the stress vs. strain is effectively independent of specimen dimensions (Bazrafshan and Stylios 2018a, b). Modulus and tensile toughness were calculated for each specimen by software; by dividing and multiplying the stress (force per unit area) to strain (proportional deformation). And the mean and standard deviation values were calculated for each group of specimens referring to modulus and tensile toughness of the samples. Modulus and tensile toughness represent their resistance to deformation and required deformation energy, respectively.

\section{Statistical analysis}

Statistical analysis was performed using a one-way analysis of variance (ANOVA) in Excel 2016 with significance set at $\mathrm{p}<0.05$. All statistical values were reported as means of standard deviation.

\section{Results and discussion}

CNCs were obtained through acid hydrolysis; the acid hydrolysis process required concentrated sulfuric acid in a very harsh reaction condition to extract the crystalline region of $\alpha$-cellulose in nanoscale and successful preparation of rod-like sulfate CNCs were observed from SEM images in which the freshly prepared CNCs had average length and diameter of $201.54 \pm 24.93$ and $19.01 \pm 5.87$, respectively. Sulfuric acid was used as it produces a negative surface charge on CNCs which leads to more stable suspensions. It is believed that sulfuric acid-treated CNCs are typically associated with sulfate ester groups (approximately $0.5-2 \%$ ) covalent bonding on the surface of CNCs (Abitbol et al. 2013; Liu et al. 2014; Börjesson and Westman 2015). For coating purposes, these anionic compounds were expected here to facilitate the coating process using a protonating solvent (FA).

The schematic illustration of this work is shown in Fig. 1a. The CNC films were prepared through typical evaporation-induced self-assembly from suspensions 
to suppose that approximately the same average thickness achieved through relatively equal solid contents under ambient conditions. The thickness of the films was $5.24 \mu \mathrm{m}$ measured individually through SEM image and ImageJ averaged from 200 dimensions. Since the optimization of the factors influencing the performance of the air-dried film was not in the scope of this work, the typical CNC films were achieved with structural irregularities resulting in mosaic-like domains under cross-polarized light, as it is in Fig. 2a.

Generally, the optical properties of CNC films are thought of as multilayer interference, which is qualitatively understood in terms that many thin layers self-assembled and the associated optical properties can be changed by a varied number of layers and self- assembled arrangements over macroscopic areas in a specimen forming birefringence effect (Kelly et al. 2013; Vignolini et al. 2013). However, due to anisotropy of the internal structure of the typical $\mathrm{CNC}$ films, their birefringent structures are perceived through POM images in multi-colors from varied distinguishable irregular domains in size, shape, and brightness in which their boundaries are in a microscopic length scale on the order of tens of microns (Saha and Davis 2018). The POM image of a selected region of the studied CNC film (Fig. 2a) is in agreement with the above-mentioned work displaying mosaic-like domains with different irregular shapes and colors such as green, blue and red. This indicates that under ambient conditions, the typical $\mathrm{CNC}$ arrangements can be shaped randomly and

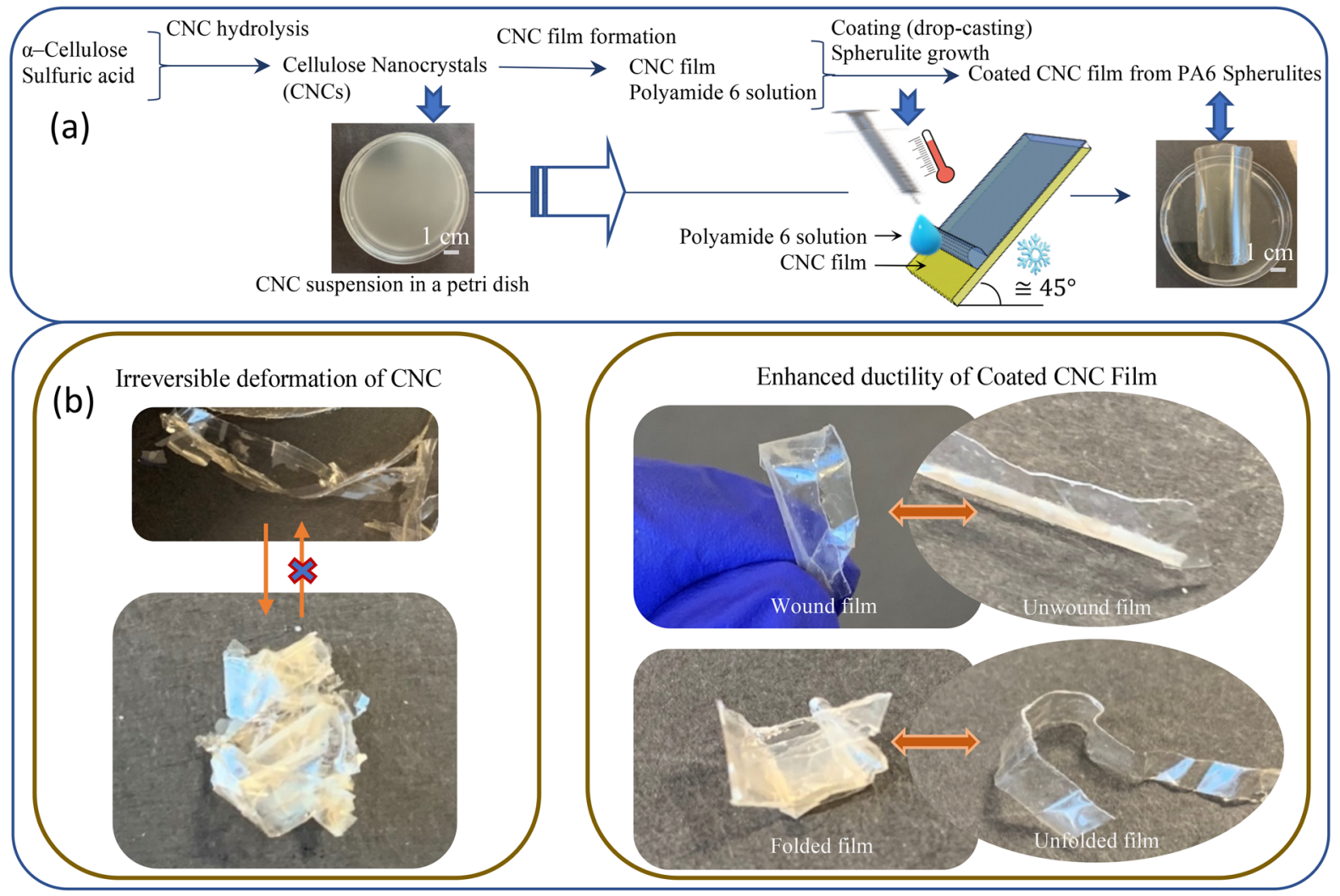

Fig. 2 a Schematic illustration of the coating process of CNC film supported by a syringe and an inclined plane tilted at an angle. b From the observational experiments on mechanical behavior (folding and winding) of a piece of $\mathrm{CNC}$ film, upon under compression/tension influenced by an external force, the CNCs film is irreversibly folded and broken down, cracks may initiate from rigid boundaries of the film structure which deduces axial splitting with limited plastic deformation. In contrast, the coated CNC film retains the integrity of the film under external mechanical force. The spherulites layer exhibits a polycrystalline microstructure when a tensional and compressive load is applied, the deformed films can be unfolded/ unwound, which means that the protecting layer of spherulites can restrain the crack to extend and provide an enhanced plastic deformation for the coated CNC film 
variously in terms of pitch lengths and helicoid tilt axes in different regions in comparison with singlepitch CNC films which shaped under specific conditions (Saha and Davis 2018; Wilts et al. 2017).

Furthermore, the studied CNC films were found brittle (Fig. 1b) and even difficult to be detached from the surface of the petri dish. This founding is similarly discovered by various research groups when they had to evaluate the sample flakes or on glass slides (Park et al. 2014; Dumanli et al. 2014b; Saha and Davis 2018). Also, according to Fig. 2b, the surface of CNC film with structural irregularities was observed uneven which was shown schematically in Fig. 2c. As in Fig. 2c, the highly irregular domains varied in size can be distinguished by darker/brighter boundaries, possibly having low solid contents that may be of weak points influencing irreversible film deformation. As mentioned earlier, eliminating or undermining the origins of the non-uniformities is not in our research scope. Instead, understanding the practical importance of functional coating in order to achieve relatively symmetrical colorful domains and reasonable mechanical strength was on purpose, which otherwise, can limit the use of selective reflection of CNC film for some applications requiring both properties together.

\section{Spherulites growth on the CNC film}

Drop-casting as a solution processing method was applied using the virtue of viscose forces. This coating method is particularly important here since it can be simply amenable to continuous, steady-state or dropon-demand printing methods and it is more likely to have no impact on thermal degradation of CNCs compared to the molten states of the coating polymeric materials. In our laboratory, relatively the same surface morphology was achieved using the dipcoating method; however, studied samples were coated through drop-casting because the surface coating of one side of samples was proposed. Here a simple binary coating system consisting of polymer (PA6) and solvent (FA, boiling point $=100.8^{\circ} \mathrm{C}$ ) was applied. Slight diffusion of FA into the CNC film was projected because of polar interactions (dipole-dipole) and its high tendency to hydrogen-bonding as a protonating agent. This interaction is important to cover imperfections of $\mathrm{CNC}$ films (e.g. uneven surfaces, pores, cracks) on the surface by flowing and simply adhering to the superficial layer of $\mathrm{CNC}$ film. Protons in the coating solution then move to CNC layers as new acceptors. And, it is more likely that this protonation from the beneath of the coating layer was an endothermic event generating an undercooling deposition and a fast drying of the coating layer which results in growing spherulites. Furthermore, since it is believed that polymeric materials crystallize as spherulites from viscous solutions at large undercooling (Crist and Schultz 2016), another consideration was performed to amplify the undercooling of the coating layer; the studied CNC films were pre-cooled and the PA6 solution was heated to $70{ }^{\circ} \mathrm{C}$. This precooling process for the $\mathrm{CNC}$ films can also solidify the trapped moisture within the $\mathrm{CNC}$ layers preventing dislocation of nonstable nanoparticles.

The Petri dishes were kept sealed before coating and this was to prevent the formation of pores on the coating layer due to excess moisture absorption on the surface of the CNC films. On the other hand, PA6 with several hydrogen-bonding sites can be attached to the polar CNC film leading to coating adhesion which is very important for interlocking of the layers.

The novel aspect of the coating approach used in the present paper is the growth of spherulites from PA6, as shown in Fig. 3. Generally, the spherical shape of spherulites allows the folding polymeric chains (lamellar) to place in helical strands radiating from nucleation points with all possible polymeric chain orientations in the plane (Crist and Schultz 2016). This leads to polycrystalline growth patterns with ordered radial symmetries and the randomization of the local crystalline orientation that accompanies crystal grain nucleation at the growth front (growth front nucleation). Although there is no generally accepted theory for spherulite crystallization for polymeric fluids, our observations suggest that the morphology of spherulites (Fig. 4) may be influenced by the uneven surface of CNC film as well as the occurrence of "secondary" nucleation at the growth front from polymeric fluids. And, the large-scale isotropy of spherulitic growth arises from the predominance of growth front nucleation in microscales. However, according to literature the upward growth direction of multi-layer spherulites (Fig. 4) may be due to a dominant drying direction as reported for other polymeric fluids (Dörling et al. 2014). The newly achieved morphology is like a 3-dimensional prismatic opal-like structure of crystals that shaped on the CNC films. Hence, PA6 crystallization follows a different process to that in chiral 

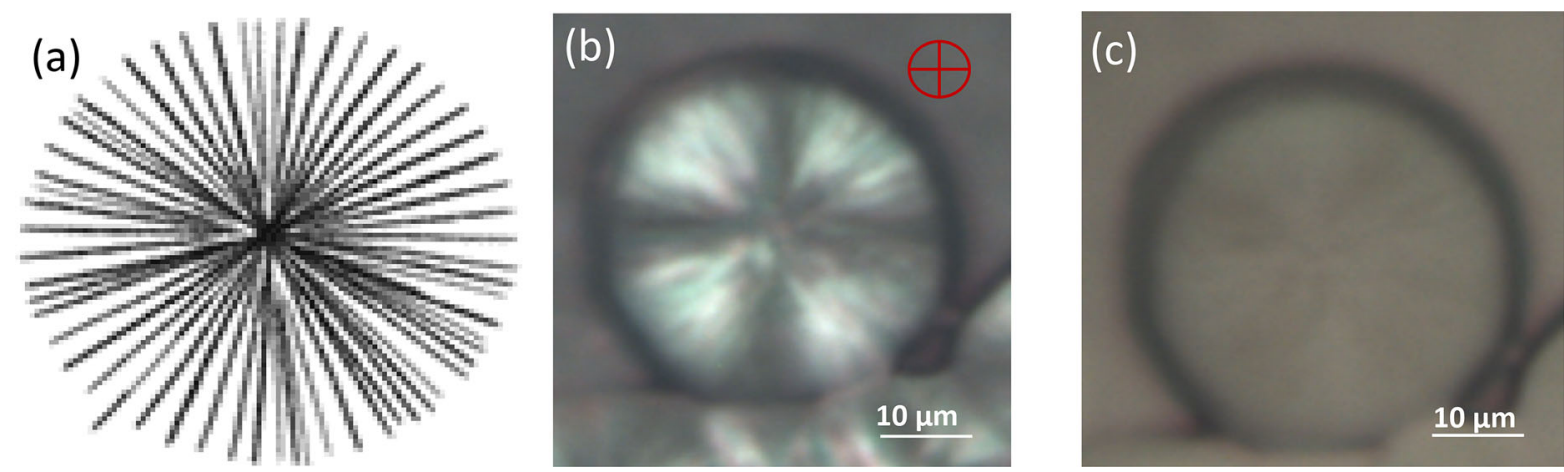

Fig. 3 a Sketches of spherulite, $\mathbf{b}$ crossed polarized image of a spherulite; the pattern is unchanged by rotating the spheruliteabout the viewing direction or by interchanging the directions of polarizer and analyzer, $\mathbf{c}$ the appearance of a spheruliteperceived without the polarizer with a mean diameter of $40.64 \pm 3.23 \mu \mathrm{m}$. Scale bars are $10 \mu \mathrm{m}$
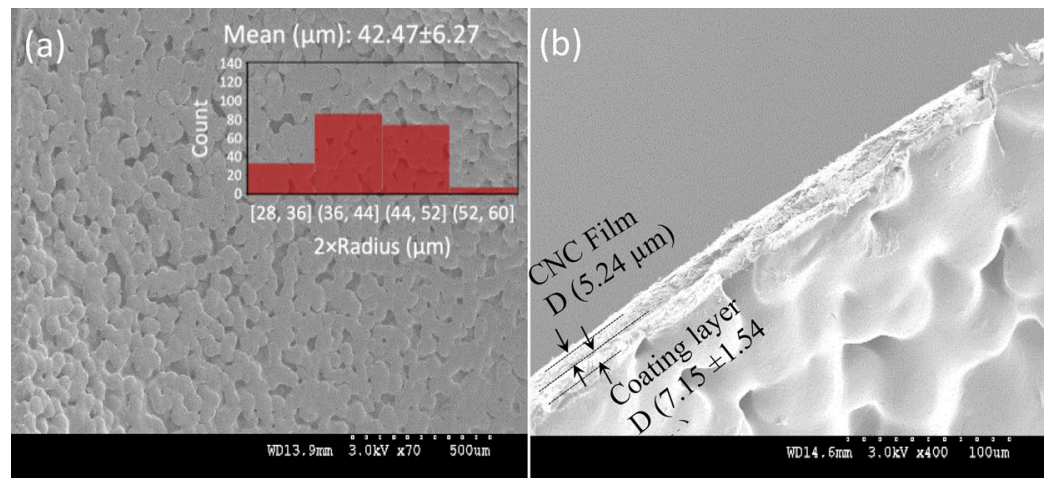

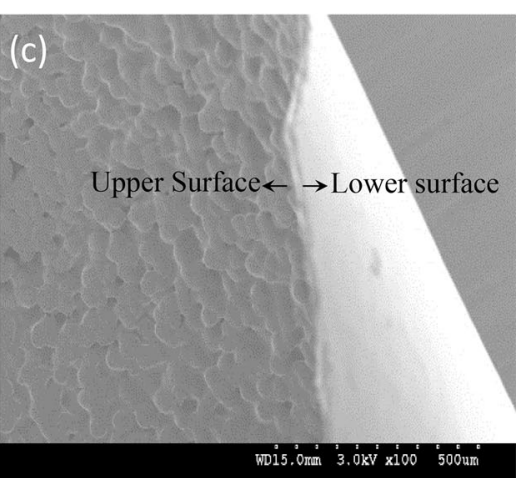

Fig. 4 Typical SEM micrographs of Coated CNC Film and grown spherulites on the upper surface with an average diameterof $42.47 \pm 6.27 \mu \mathrm{m}$ (a), with an average thickness of

photonic CNC films, spherulites produce different crystalline morphology and configurations, Figs. 3 and 4.

Due to the above-mentioned intermolecular and interfacial interactions, the coated CNC films without rips and tears were readily detached across the petri dish for further investigations (Fig. 1a). From the microscopic observations (Fig. 5a, b), the diffusion of the solvent through the substrate had no or negligible effect on the color-structure of the CNC films without possibly moving the $\mathrm{CNCs}$ arrangements

Optical anisotropy and radially symmetric microstructure of PA6 spherulite

To achieve more in-depth insight into the optical pattern of the crystalline feature of spherulites, a glass slide was coated with PA6 and then microstructures of
$7.15 \pm 1.54 \mu \mathrm{m}$ (b) and the patterned upper surface of Coated CNC film inwhich the lower side (c) is uncoated as shaped while drying

individually grown PA6 crystal were viewed between crossed polarizers. According to the optical microscope imaging, birefringence and extinction domains, and so-called Maltese cross pattern were observed in the regular and uniform circular symmetrical pattern of the individual PA6 spherulite, as in Fig. 4b, c. And, Like some other polymeric spherulites that were reported in literature, the internal anisotropic structure of these banded spherulites, which induced from polymeric chain orientations in the plane, is detected through (a) the light transmission when the polymeric chains have a non-zero angle with both polarizers; or (b) zero extinction band when the direction of the chains overlaps with one of the crossed-polarizers (Dingler et al. 2019; Crist and Schultz 2016). This optical transmission- extinction bands allow each banded PA6 spherulite perceived relatively symmetrical on the surface and boundaries. 

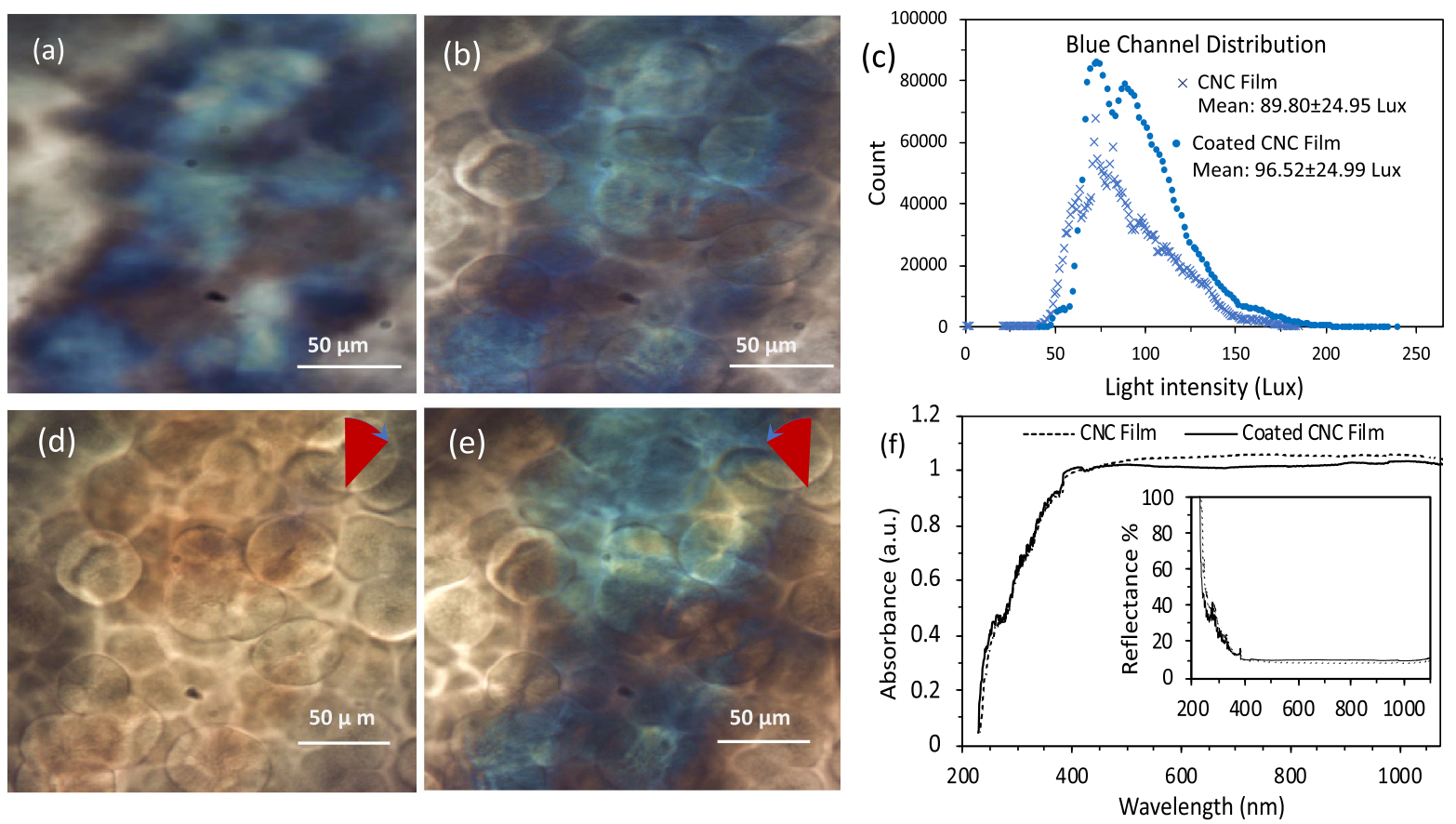

Fig. 5 a and $\mathbf{b}$ A representation of a microscopic scale of CNC film and coated CNC film observed through crossed polarizers, in which considerable change was perceived due to the presence of spherulites of PA6 covering the CNC film. c Based on the color histogram of the blue broadband information, the bright blue color of the coated CNC film (b) is more intense than

\section{Optical performance of the coated CNC film}

The CNC film showed a low diffusion reflection of up to $10 \%$ and low light absorption in the visible and near-IR regions, as in Fig. 5f. When surface coating, the maximum of the light absorption slightly drops to reach a lower value and the reflection increased insignificantly by less than $1 \%$. This gives us evidence that diffusion reflectance of the coated surface is not noticeable, and the thickness of the coating layer is not to significantly obstruct the transmitted light to lower layers and vice versa. Furthermore, this amenable behavior even tends to continue the CNC film trend in the ultraviolet region.

A colorful domain of CNC film with a distinguishable size of dark regions (Fig. 5a) was specifically considered to identify the impact of spherulite formation on the intensity of transmitted rays (brightness) through this domain. In Fig. 5a, different shapes of colorful regions are visible that are varied in intensity; large dark regions characterize situations where that of CNC film without coating layer (a). $\mathbf{d}$ and $\mathbf{e}$ the feature of spherulites were observed invariant under rotation of the polarizer. fight absorption-reflection from UV-vis spectrophotometry showing that the coating spherulites have an insignificant impact on high transparency of the thin CNC film

incident polarized light is blocked (extinction domain) and not to transmit when the analyzer is arranged perpendicular to the polarizer (Kose et al. 2019). This represents the highly nonuniform optical anisotropy of the observed domain under polarized light. Again, non-uniformities in the initial CNC dispersions and microstructural changes during drying typically result in highly irregular chiral photonic regions which is normal of any uncontrolled self-assembly method and repeatedly reported in literature (Kose et al. 2019; Saha and Davis 2018).

Figure $5 \mathrm{~b}$ shows the same colorful domain of CNC film, which was observed after the coating from PA6 spherulites. The existing colors (e.g., blue colors) in the coated CNC film reveal that (a) the CNC arrangements are not affected by the employed coating process and crystallization, and (b) the bands of spherulites as coating layer occur depending on the incident light wavelength that transmitted through the CNC film. However, due to the occurrence of both (scattered) reflection and (scattered) refraction within 
the internal structure of spherulites, the coated CNC film is perceived with different transmission and zero (extinction) bands while the intensity of the transmitted rays do not remain constant throughout each spherulite. The maltese cross is not clearly observed on the surface; this can be due to the non-evenness of the substrate (CNC film surface) as well as the multilayer orientation of the spherulites preventing the direction of the internal chains to regionally overlap with one of the polarizers.

The interaction of transmitted rays within each spherulite provide information in two ways: (a) dark round edges of spherulites; the main color (e.g. the wavelength of blue light) of the direct image formed by $\mathrm{CNC}$ film subjected to reflection/refraction being observed in a darker color on edges surrounded by a bright halo and (b) rayleigh scattering of the surface; the transmission depending on the wavelength may be scattered when the internal orientation of spherulites have a non-zero angle with both polarizers. Consequently, due to the large average size of the spherulites $(42.47 \pm 6.27 \mu \mathrm{m})$ and all unique optical phenomenon that may happen on transmitting wavelengths, the light transmission-extinction bands of the $\mathrm{CNC}$ film are influenced and perceived through radial symmetry of illuminated spherulites which are also responsible for the formation of circular extinction patterns for the coated CNC film.

This can be also concluded that the spherulites are illumination angular responsive crystals here and the angle of transmitting rays symmetrically may be further altered to a greater or lesser angle. This simply means that the coating spherulites may change intensity distribution of transmitted rays of the CNC film; the brightness of the refracted ray may be perceived 'decreased', simultaneously the brightness of the reflected ray may be perceived 'increased' and vice versa. To compare the intensity distribution of transmitted rays of CNC film and the coated CNC film, the brightness calculation was performed using RGB digital image processing of ImageJ software. And, the associated color histogram showing blue channel distribution was revealed in Fig. 5c. The average brightness of the coated CNC film (96.52 Lux) is at a higher intensity comparing to that of the naked CNC film (89.80 Lux). And, this apparent upshifting in brightness, which simply indicates a color shift to a bluer shade and lesser blackness, may be more appropriate for many applications. Also, the characteristic spherulites are observed invariant under rotation of the polarizer, Fig. $5 \mathrm{e}$, f.

To more in-depth study the effect of spherulites on the appearance of a brighter and larger mosaic domain of the CNC film, the studied films were individually placed on a polystyrene sheet. Crossed-polarized optical microscopy reveals that this placement selectively reflects left-hand circularly polarized light at a specific set of wavelengths (Dumanli et al. 2014b). This led to higher intensity and more diversity of perceived light rays, hence the samples were observed brighter and more colorful, as it is in Fig. 6a, b. According to Figures $7 \mathrm{~b}$ and $\mathrm{d}$, the radial symmetry of banded spherulites revealed relatively with the same appearance as seen in Fig. 5b. And, by relatively regular shape and size of banded spherulites and repetitively changing the color brightness (referring to the predictable intensity distribution between transmission and extinction bands of each spherulite), the irregular domains of the $\mathrm{CNC}$ film, which were placed beneath the local spherulites, have been restricted within the surface area of each spherulite and positively corrected; from the binary image (Fig. 6c), digitally processed by ImageJ, the grayscale areas (darkness) of CNC film were visually reduced in a smaller surface area by banded spherulites.

Furthermore, with respect to the relatively similar shape and size of the banded spherulites (Figs. 5a and $7 b)$, reproducibility of the regular polycrystalline growth of the banded spherulites is more certain comparing to the irregular transmission-extinction bands of the chiral photonic CNC films. The coating layer here may be like a polishing layer that reduce the optical imperfections and improve the overall intensity of the transmitted light rays, simultaneously. And, this new outstanding appearance that was introduced through banded spherulites to the CNC film may happen similarly on photonic $\mathrm{CNC}$ domains that require similar and predictable extinction bands in the microscopic study.

Physiochemical properties of the coated CNC film

As shown in Fig. 7a, the FTIR spectrum of the CNC film presented distinguishing $\mathrm{CNC}$ absorption peaks, a stretching band at $3422 \mathrm{~cm}^{-1}$ and $2895 \mathrm{~cm}^{-1}$ corresponding to the hydroxyl group $\mathrm{O}-\mathrm{H}$ and the $\mathrm{C}-\mathrm{H}$ bond, respectively; a weak band at $1635 \mathrm{~cm}^{-1}$, originating typically from absorbed humidity and the 


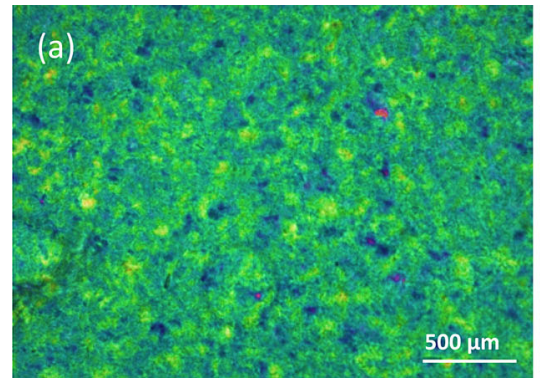

Fig. 6 POM images of a CNC film with irregular domains on polystyrene sheet (a) and a coated CNC film (b) in which each PA6 spherulite provides a radially symmetric structure for CNC films. From the binary image, $50 \%$ black and white threshold (c1 and 2) digitally processed by ImageJ, the grayscale areas of

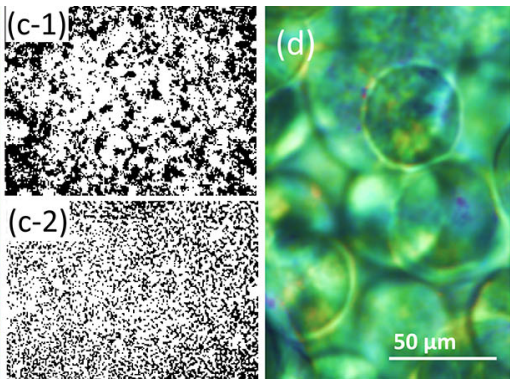

CNC film (c-1) were visually reduced and perceived in a smaller surface area through spherulite crystallization (c-2). POM image of a larger magnitude of the coated CNC film referring to darker domains of spherulites consisting of the curved boundaries (d)
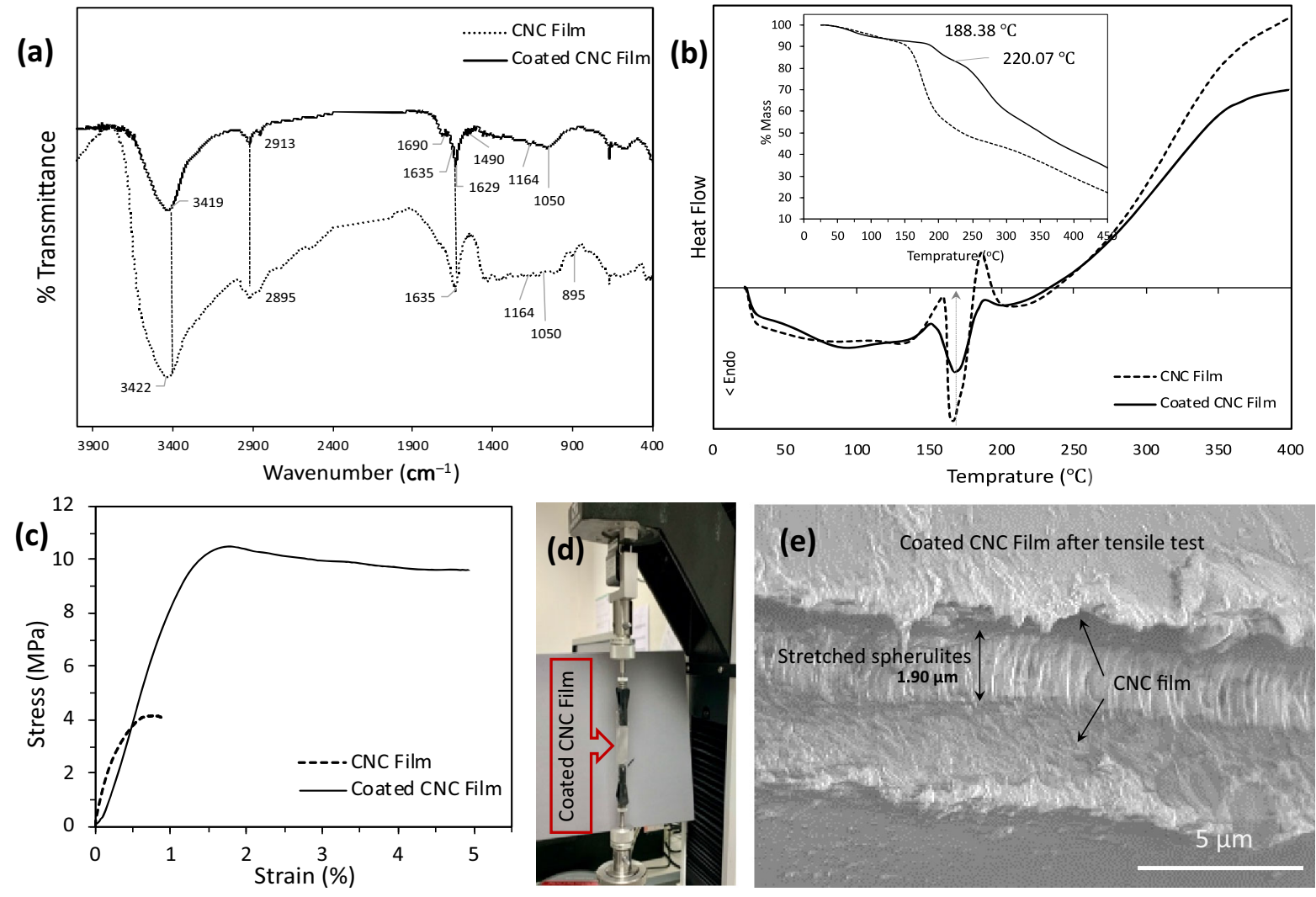

Fig. 7 Typical physiochemical properties of the samples: CNC Film and Coated CNC Film, molecular structure from FTIR (a), Thermal behavior from DSC and TGA analysis (b), the typical stress-strain curve from the tensile test (c); an image of a

absorption band at 1050 and $895 \mathrm{~cm}^{-1}$, which was allocated to the deformation of the $\mathrm{C}-\mathrm{H}$ bond of $\beta$ glycosidic linkages between glucose (Tang et al. 2013). The absorption peak between $1050 \mathrm{~cm}^{-1}$ and specimen that was held between jaws of Instron Tensile Tester applying load until it breaks (d) and an SEM image of stretched coated CNC film (e). captured after the tensile test

$1160 \mathrm{~cm}^{-1}$ was due to the stretching of the $\mathrm{C}-\mathrm{O}$ bond, which is ascribed to the ether main bonds. Stretching $\mathrm{S}=\mathrm{O}$ was somewhere between 1510 and $1380 \mathrm{~cm}^{-1}$. From the FTIR analysis of the coated CNC film, apart 
from Amide absorption peaks of PA6, the stretching band at $3422 \mathrm{~cm}^{-1}$ corresponding to the hydroxyl group $\mathrm{O}-\mathrm{H}$ received a shifting to a lower wavelength $\left(3419 \mathrm{~cm}^{-1}\right)$ that is important and is attributed to the strengthening of the hydrogen bonds at the local surface of superficial layer, as well as ordering and strains of PA6 chains induced by the spherulites growth process which shifted the corresponding peak of the hydroxyl group.

From the thermal analysis of CNC films (Fig. 7b), the sulfuric acid-treated CNCs were sensitive to heat, as expected. At temperatures above $100{ }^{\circ} \mathrm{C}$, the $\mathrm{CNCs}$ underwent desulfation; afterward the helical and chiral of CNCs were destroyed because of intense desulfation at $168{ }^{\circ} \mathrm{C}$ leading to the separation of the anionic sulfate groups from the surface of the CNCs.

The thermal behavior of the coated CNC film was also evaluated. According to Fig. 7b, the DSC analysis of the coated CNC film reveals that the intensity of the desulfation peak decreased. This can be due to an exothermic procedure that simultaneously occurred in PA6 spherulitic layers e.g., recrystallization. However, some CNC layers experienced degradation at $168^{\circ} \mathrm{C}$ likewise the $\mathrm{CNC}$ film. The thermal degradation was also examined through TGA analysis, in which the decomposition event partially happened at a higher temperature $\left(>168{ }^{\circ} \mathrm{C}\right)$ as shown in the same graph, Fig. 7a. This enhanced thermal stability can be due to the strong intermolecular interactions including hydrogen bonding that are characterized by the spherulite coating. Also, the coating spherulites may strengthen heat transfer phenomena leading to gradually decomposition event of CNCs.

Through the tensile test, we explored the response of the symmetrical polymeric chain orientations of spherulites and their associated free volume under externally applied stress. Figure $7 \mathrm{c}$ shows a typical stress-strain curve for coated CNC film whereas the corresponding curve for $\mathrm{CNC}$ film is also present as the reference. For testing the mechanical behavior of the samples, each specimen $(n=5$ of each sample) was held between the jaws of Instron Tensile Tester applying load until it breaks (Fig. 7d).

The tensile toughness and modulus were calculated for each specimen through the software connected to the machines and their mean values with the correlated standard deviation were calculated for each group of specimens referring to the tensile toughness and modulus of the samples.
According to Table 1, the PA6 spherulites provide an enhanced mechanical strength for the coated CNC film under load and strain, comparing to the pristine CNC film. From Fig. 7d, it is possible that the antiparallel chain orientation of PA6 spherulites can drive elongation of films in a direction parallel to the increasing strain axis which is ended up with stretched chains in the direction of strain axis. This behavior led to more than five time enhancement of elasticityplasticity strength under stress-strain deformation. And at a crystalline scale, this mechanical reinforcement that induced from spherulites can be explained as follows. The antiparallel chain orientation of spherulites may entangle dislocations and prevent polymeric chains (interlamellar) from sliding providing an improvement of the elasticity behavior to the coated CNC film up to the yield of strength, at the point of $9.86 \mathrm{MPa}-1.73 \%$ on stress-strain curve, Fig. $7 \mathrm{c}$. As further tensile load is applied, spherulites turn gradually from an ordered chain orientation to a disordered and then stretched pattern, and simultaneously the cracks may appear and run through the CNC layers as shown in the SEM image of Fig. 7e. This can be a consequence of irreversible deformation of PA6 chains within the internal structure of spherulites in this stage when a load-strain over the yielding point is applied. Plastic deformation that leads to fracture happened at approximate stress and strain of $9.62 \mathrm{MPa}$ and $4.95 \%$, respectively, when the overextended PA6 chains were unable to support any load. The growth of critical cracks subject to cyclic stresses due to the fatigue of $\mathrm{CNC}$ films was not investigated in this study.

\section{Conclusions}

A typical air-dried CNC film was prepared from suspensions. The CNC film was then coated with PA6 under certain conditions in which the PA6 chain orientations had the same sense revealing spherulites. The effect of spherulite crystallization on irregular chiral photonic domains of CNC film was investigated. The spherulites exhibited relatively symmetrical round shapes grown on the top layer of the CNC film. The coating layer was not to obstruct the transmitting light to the CNC film. Under polarized light, the light transmission-extinction bands of CNC 
Table 1 Mechanical properties of the samples

\begin{tabular}{lllllll}
\hline Samples & $\begin{array}{l}\text { Breaking stress } \\
(\mathrm{MPa})^{\mathrm{a}}\end{array}$ & $\begin{array}{l}\text { Breaking strain } \\
(\mathrm{mm} / \mathrm{mm}) \%^{\mathrm{a}}\end{array}$ & $\begin{array}{l}\text { Tensile stress } \\
(\mathrm{MPa})^{\mathrm{b}}\end{array}$ & $\begin{array}{l}\text { Tensile strain } \\
(\mathrm{mm} / \mathrm{mm}) \%^{\mathrm{c}}\end{array}$ & $\begin{array}{l}\text { Tensile toughness } \\
\left(\mathrm{cJ} . \mathrm{mm}^{-3}\right)^{\mathrm{b}}\end{array}$ & $\begin{array}{l}\text { Modulus } \\
(\mathrm{MPa})^{\mathrm{b}}\end{array}$ \\
\hline CNC Film & $4.07 \pm 0.17$ & $0.92 \pm 0.09$ & $4.16 \pm 0.40$ & $0.84 \pm 0.12$ & $0.34 \pm 0.03$ & $341.06 \pm 15.78$ \\
$\begin{array}{l}\text { Coated } \\
\text { CNC Film }\end{array}$ & $9.62 \pm 0.36$ & $4.95 \pm 0.11$ & $9.86 \pm 0.33$ & $1.73 \pm 0.36$ & $1.83 \pm 0.22$ & $593.65 \pm 9.02$ \\
\hline
\end{tabular}

(a) $(p=0.001)$

(b) $(p<0.01)$

(c) $(p<0.003)$

film were perceived through the illuminated spherulitic microstructures. Due to all unique optical phenomena that may happen on transmitting light rays and regular extinction band of banded spherulites, a relatively ordered optical appearance containing radial symmetries was devoted to the optical irregularities of the CNC film. Besides, the mechanical strength of the coated CNC film was enhanced by the spherulites providing tension resistance. Due to the presence of coating spherulites, the thermal decomposition of CNCs was segmented leading to a delay in the desulfation of a portion of CNCs. Since a large variety of polymers is available, these morphological patterns allow for the development of a variety of portable functional assemblies in photonic sensing. improve the overall intensity of the transmitted light rays ordering effect of their discrete local and orientation positively influenced the effects of structural non-uniformities of the typical CNC films. Since polycrystalline growth of spherulites may occur in the same way from other semicrystalline polymers under their thermodynamic conditions, this surface design and coating approach is suggested for the protection and the future development of a variety of portable functional assemblies of CNC films for photonic sensing requiring enhanced performance in optical and mechanical properties.

Acknowledgments The author is grateful to Profs. George Stylios, Fiona Waldron and Julian Malins for discussions and comments in relation to this manuscript.

Open Access This article is licensed under a Creative Commons Attribution 4.0 International License, which permits use, sharing, adaptation, distribution and reproduction in any medium or format, as long as you give appropriate credit to the original author(s) and the source, provide a link to the Creative Commons licence, and indicate if changes were made. The images or other third party material in this article are included in the article's Creative Commons licence, unless indicated otherwise in a credit line to the material. If material is not included in the article's Creative Commons licence and your intended use is not permitted by statutory regulation or exceeds the permitted use, you will need to obtain permission directly from the copyright holder. To view a copy of this licence, visit http://creativecommons.org/licenses/by/4.0/.

\section{References}

Abitbol T, Kloser E, Gray DG (2013) Estimation of the surface sulfur content of cellulose nanocrystals prepared by sulfuric acid hydrolysis. Cellulose 20:785-794. https://doi. org/10.1007/s10570-013-9871-0

Alanis A, Valdés JH, Guadalupe NVM, Lopez R et al (2019) Plasma surface-modification of cellulose nanocrystals: a green alternative towards mechanical reinforcement of ABS. RSC Adv 9:17417-17424. https://doi.org/10.1039/ C9RA02451D

Bazrafshan Z, Stylios G (2018a) High performance of covalently grafting onto collagen in the presence of graphene oxide. Nanomaterials 8:703. https://doi.org/10.3390/ nano8090703

Bazrafshan Z, Stylios GK (2018b) Custom-built electrostatics and supplementary bonding in the design of reinforced Collagen-g-P(methyl methacrylate-co-ethyl acrylate)/ nylon 66 core-shell fibers. J Mech Behav Biomed Mater 87:19-29. https://doi.org/10.1016/j.jmbbm.2018.07.002

Bazrafshan Z, Stylios GK (2019) A novel approach to enhance the spinnability of collagen fibers by graft polymerization. Mater Sci Eng C 94:108-116. https://doi.org/10.1016/j. msec.2018.09.016

Bondeson D, Mathew A, Oksman K (2006) Optimization of the isolation of nanocrystals from microcrystalline cellulose by acid hydrolysis. Cellulose 13:171. https://doi.org/10. 1007/s10570-006-9061-4

Börjesson M, Westman G (2015) Crystalline nanocellulosepreparation, modification, and properties. Cellulose-fundamental aspects current trends, pp 159-191. https://doi. org/10.5772/61899 
Crist B, Schultz JM (2016) Polymer spherulites: a critical review. Prog Polym Sci 56:1-63. https://doi.org/10.1016/j. progpolymsci.2015.11.006

Diddens I, Murphy B, Krisch M, Müller M (2008) Anisotropic elastic properties of cellulose measured using inelastic X-ray scattering. Macromolecules 41:9755-9759. https:// doi.org/10.1021/ma801796u

Dingler C, Dirnberger K, Ludwigs S (2019) Semiconducting polymer spherulites-From fundamentals to polymer electronics. Macromol Rapid Commun 40:1800601. https://doi.org/10.1002/marc.201800601

Dörling B, Vohra V, Dao TT et al (2014) Uniaxial macroscopic alignment of conjugated polymer systems by directional crystallization during blade coating. J Mater Chem C 2:3303-3310. https://doi.org/10.1039/C3TC32056A

Dumanli AG, Kamita G, Landman J et al (2014a) Controlled, bio-inspired self-assembly of cellulose-based chiral reflectors. Adv Opt Mater 2:646-650. https://doi.org/10. 1002/adom.201400112

Dumanli AG, Kooij HM, Kamita G et al (2014b) Digital color in cellulose nanocrystal films. ACS Appl Mater Interfaces 6:12302-12306. https://doi.org/10.1021/am501995e

Eggleton BJ, Luther-Davies B, Richardson K (2011) Chalcogenide photonics. Nat Photon 5:141. https://doi.org/10. 1038/nphoton.2011.309

Habibi Y, Heim T, Douillard R (2008) AC electric field-assisted assembly and alignment of cellulose nanocrystals. J Polym Sci B Polym Phys 46:1430-1436. https://doi.org/10.1002/ polb.21479

Kelly JA, Yu M, Hamad WY, MacLachlan MJ (2013) Large, crack-free freestanding films with chiral nematic structures. Adv Opt Mater 1:295-299. https://doi.org/10.1002/ adom. 201300015

Kose O, Tran A, Lewis L et al (2019) Unwinding a spiral of cellulose nanocrystals for stimuli-responsive stretchable optics. Nat Commun 10:510. https://doi.org/10.1038/ s41467-019-08351-6

Liu D, Wang S, Ma Z et al (2014) Structure-color mechanism of iridescent cellulose nanocrystal films. RSC Adv 4:39322-39331. https://doi.org/10.1039/C4RA06268J

Liu P, Guo X, Nan F et al (2017) Modifying mechanical, optical properties and thermal processability of iridescent cellulose nanocrystal films using ionic liquid. ACS Appl Mater Interfaces 9:3085-3092. https://doi.org/10.1021/acsami. 6b12953

Park JH, Noh J, Schütz C et al (2014) Macroscopic control of helix orientation in films dried from cholesteric liquidcrystalline cellulose nanocrystal suspensions. ChemPhysChem 15:1477-1484. https://doi.org/10.1002/cphc. 201400062

Peng BL, Dhar N, Liu H, Tam K (2011) Chemistry and applications of nanocrystalline cellulose and its derivatives: a nanotechnology perspective. Can J Chemical Eng 89:1191-1206. https://doi.org/10.1002/cjce.20554

Saha P, Davis VA (2018) Photonic properties and applications of cellulose nanocrystal films with planar anchoring. ACS Appl Nano Mater 1:2175-2183. https://doi.org/10.1021/ acsanm. 8 b00233
Samir MASA, Alloin F, Sanchez JY, Dufresne A (2004) Cellulose nanocrystals reinforced poly (oxyethylene). Polymer 45:4149-4157. https://doi.org/10.1016/j.polymer. 2004.03.094

Sheltami RM, Abdullah I, Ahmad I et al (2012) Extraction of cellulose nanocrystals from mengkuang leaves (Pandanus tectorius). Carbohyd Polym 88:772-779. https://doi.org/ 10.1016/j.carbpol.2012.01.062

Sinko R, Mishra S, Ruiz L et al (2013) Dimensions of biological cellulose nanocrystals maximize fracture strength. ACS Macro Lett 3::64-69. https://doi.org/10.1021/mz400471y

Tang L, Huang B, Yang N et al (2013) Organic solvent-free and efficient manufacture of functionalized cellulose nanocrystals via one-pot tandem reactions. Green Chem 15:2369-2373. https://doi.org/10.1039/C3GC40965A

Tsui KH, Lin Q, Chou H et al (2014) Low-cost, flexible, and self-cleaning 3D nanocone anti-reflection films for highefficiency photovoltaics. Adv Mater 26:2805-2811. https://doi.org/10.1002/adma.201304938

Viet D, Beck-Candanedo S, Gray DG (2007) Dispersion of cellulose nanocrystals in polar organic solvents. Cellulose 14:109-113. https://doi.org/10.1007/s10570-006-9093-9

Vignolini S, Moyroud E, Glover BJ, Steiner U (2013) Analysing photonic structures in plants. J R Soc Interface 10:9. https:// doi.org/10.1098/rsif.2013.0394

Wilts B, Dumanli A, Middleton R et al (2017) Chiral optics of helicoidal cellulose nanocrystal films. APL Photon 2:040801. https://doi.org/10.1063/1.4978387

Xu M, Li W, Ma C et al (2018) Multifunctional chiral nematic cellulose nanocrystals/glycerol structural colored nanocomposites for intelligent responsive films, photonic inks and iridescent coatings. J Mater Chem C 6:5391-5400. https://doi.org/10.1039/C8TC01321G

Yang Y, Chen M, Li H, Li H (2018) The degree of crystallinity exhibiting a spatial distribution in polymer films. Eur Polymer J 107:303-307. https://doi.org/10.1016/j. eurpolymj.2018.08.041

Yao K, Meng Q, Bulone V, Zhou Q (2017) Flexible and responsive chiral nematic cellulose nanocrystal/poly (ethylene glycol) composite films with uniform and tunable structural color. Adv Mater 29:1701323. https://doi.org/10. 1002/adma.201701323

Zhang YP, Chodavarapu VP, Kirk AG, Andrews MP (2013) Structured color humidity indicator from reversible pitch tuning in self-assembled nanocrystalline cellulose films. Sens Actuators B 176:692-697. https://doi.org/10.1016/j. snb.2012.09.100

Zhu Q, Yao Q, Sun J et al (2019) Stimuli induced cellulose nanomaterials alignment and its emerging applications: A review. Carbohyd Polym. https://doi.org/10.1016/j. carbpol.2019.115609

Publisher's Note Springer Nature remains neutral with regard to jurisdictional claims in published maps and institutional affiliations. 